\title{
Prospective Photovoltaic-Thermoelectric Hybrid System
}

\author{
Gao Min*
}

Received date: 05 December 2021; Accepted date: 05 January 2021

Article type: Personal Account

Photovoltaic-thermoelectric (PV-TE) hybrid system is an appealing idea due to its potential to harvest thermal energy produced in photovoltaic conversion process or mitigate the temperature effect through cooling. The rationale for developing PV-TE hybrid system is illustrated in Fig. 1, which depicts three possible strategies that might lead to a higher overall efficiency of the hybrid system than that of photovoltaic system alone. In strategy (a), thermoelectric device is used as a cooler that is in direct contact with the solar cells. Since the solar cells exhibit negative temperature coefficient, ${ }^{[1]}$ reducing the operating temperature of the solar cells could lead to an increase in the power output of the solar cell. In strategy (b), thermoelectric device is arranged in similar manner as strategy (a) but used as a thermoelectric generator. Thermalisation loss ${ }^{[2]}$ inside solar cells will lead to an increase in the temperature of the solar cells, which in turn establishes a temperature difference across the thermoelectric generator. As a result, the waste thermal energy from the solar cell can be utilised by thermoelectric generator to convert into useful electric power. In strategy (c), the solar energy in infrared spectrum, which has no use to solar cells, is separated from the visible spectrum and directed to a thermoelectric generator for power generation. ${ }^{[3]}$ Clearly, these strategies are built on the sound scientific rationales. The key question is if they will lead to a realistic improvement when other influences are taken into consideration. This paper aims to offer critical assessments on the prospect in these research directions.

\section{Hybrid system with thermoelectric cooling}

Thermoelectric device can be used as a cooler to reduce the operating temperature of solar cell as shown in Fig. 1(a). Since most solar cells have a negative temperature coefficient, the power output of the solar cells will increase if their operating temperature is reduced. However, thermoelectric device requires power to produce cooling. ${ }^{[4]}$ Consequently, the benefit of the hybrid system depends on whether the power gained

School of Engineering, Cardiff University, United Kingdom.

*Email: min@cardiff.ac.uk (G. Min) from the solar cell overcompensates the power consumed by the thermoelectric device. The feasibility of such hybrid systems is being investigated by examining the temperature change in relation to the coefficient-of-performance (COP) of the thermoelectric devices. Fig. 2 shows the COP of thermoelectric device under different operating modes. When operating in the Peltier mode, the temperature of the solar cell is decreased below the temperature of heat sink at expense of large power consumption by thermoelectric device. On the other hand, the COP is much larger when operating in the heat pump mode, where the temperature of solar cell is higher than (or equal to) the temperature of heat sink. A more interesting operation is self-powered heat pumping mode, ${ }^{[5]}$ where the thermoelectric device does not require input power. The Peltier cooling takes place when the terminals of thermoelectric device are short-circuited. In this case, the thermoelectric device operates in both the cooling and generation mode.

Theoretical analysis has been carried out to assess whether such PV-TE hybrid systems can lead to an increase in overall efficiency. Table 1 shows the results of a case study carried out based a typical low concentration case, where the cooling of solar cell is needed. The net power gain was determined from the power gain in the solar cell and the power loss in the thermoelectric device. It can be seen from table that the net power gain for all 4 cases is negative. This indicates that the cooling of the solar cell using thermoelectric device cannot lead to an increase in the overall efficiency of the hybrid system because the power consumed by thermoelectric device is always more than the power gained from the solar cells. Although the results in Table 1 are obtained based on a specific case, it can be shown rigorously that it is impossible to obtain a net gain of the power by cooling the solar cell using thermoelectric devices (see Appendix). Nevertheless, it is worth mentioning that such hybrid system can be used as a convenient experimental setup for studying the temperature dependence of solar cells. It may also be employed to improve the stability of solar cells at small expense of efficiency reduction. 


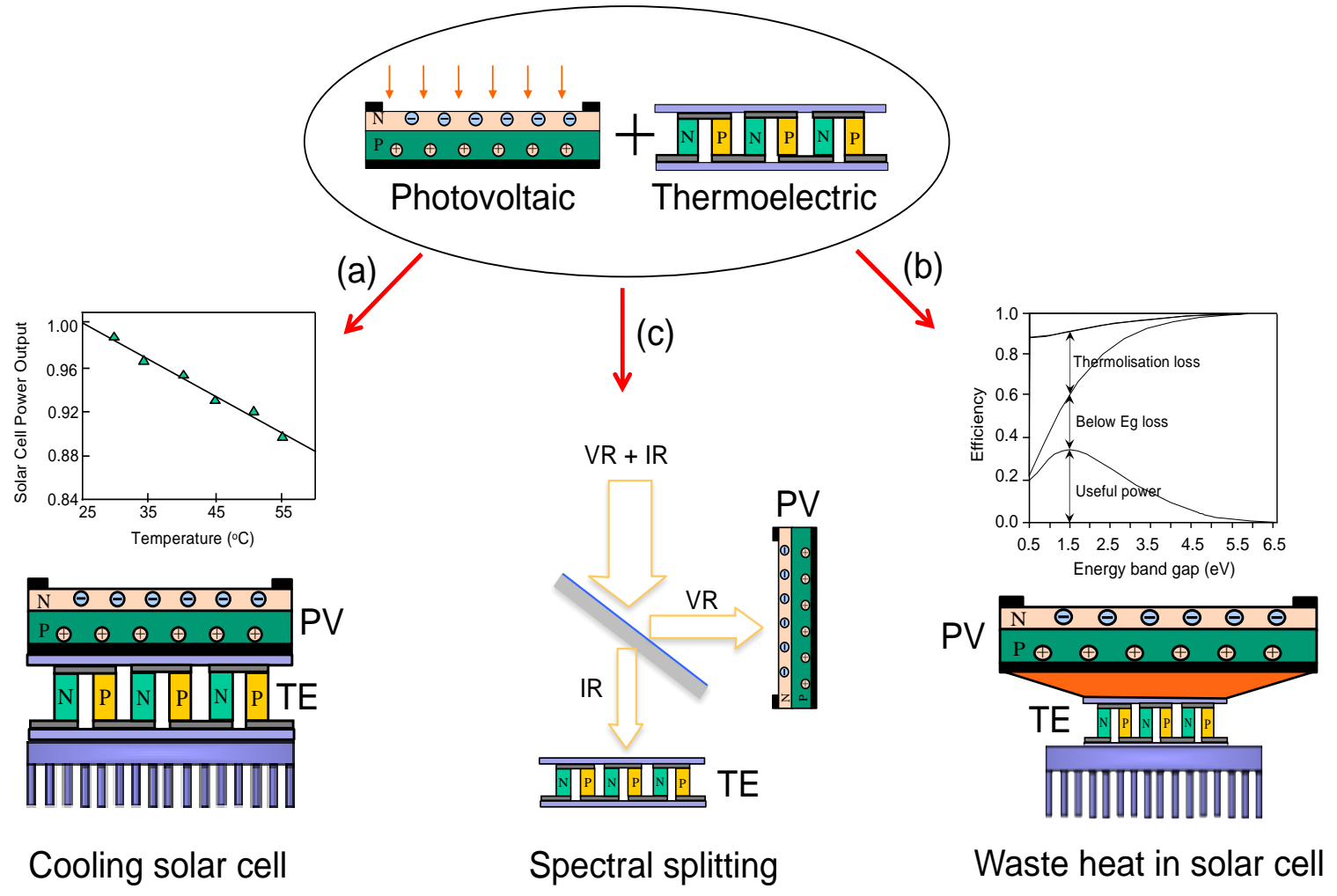

Fig. 1 Schematics of three PV-TE hybrid systems a) thermoelectric device in cooling mode to control the operating temperature of the solar cell; b) thermoelectric device in generation mode converting waste heat in solar cell into electricity; c) spectral splitting to facilitate the photovoltaic conversion using photons in visible spectrum and thermoelectric harvesting using thermal energy in infrared spectrum.

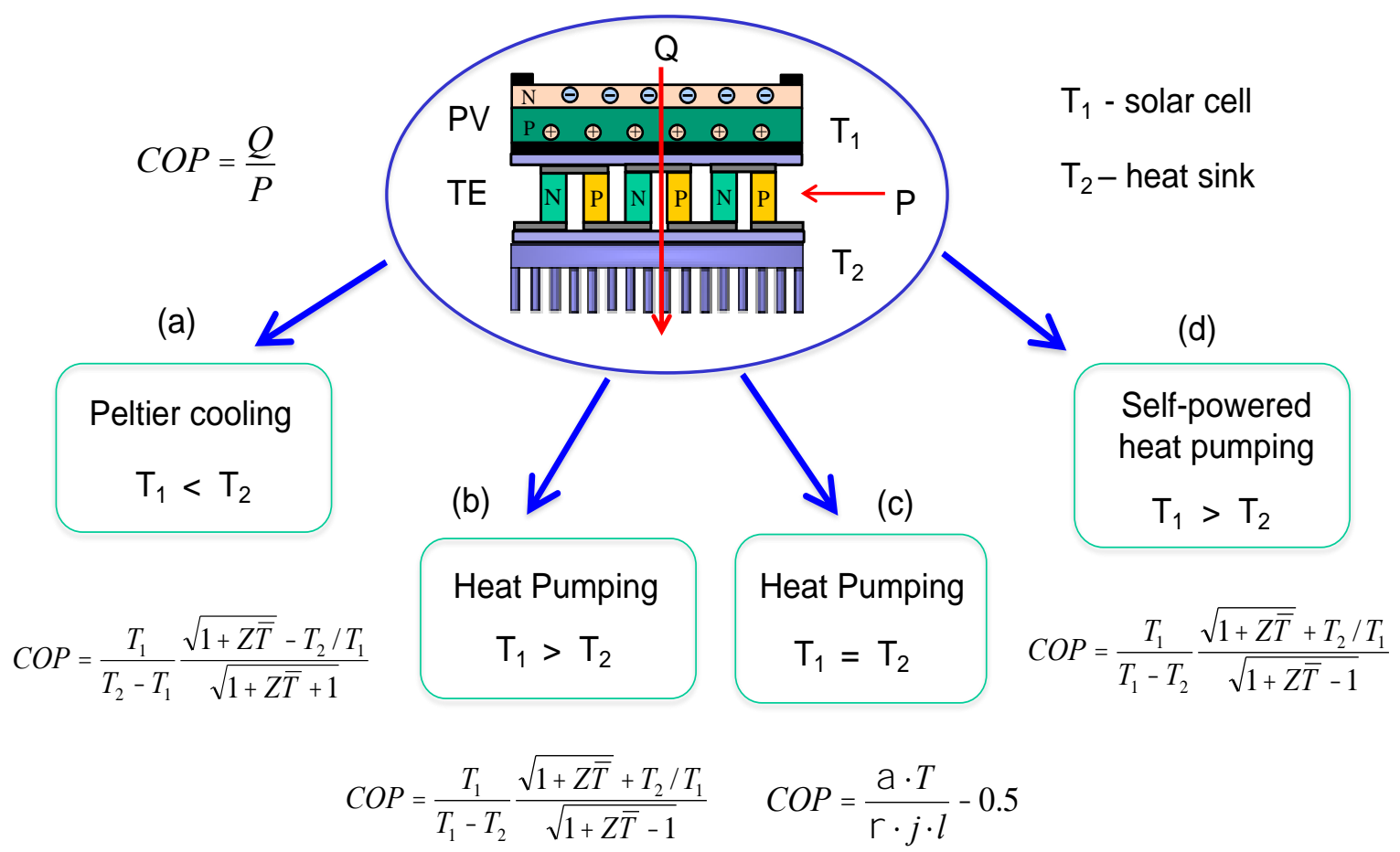

Fig. 2 The coefficient-of-performance (COP) of thermoelectric cooling, where $T_{1}$ and $T_{2}$ are the temperature of solar cell and heat sink, respectively. $Z \bar{T}$ is the dimensionless figure-of-merit of thermoelectric device, $\alpha$ the Seebeck coefficient, $\rho$ the electrical resistivity, $j$ the current density through the thermoelectric leg, and $l$ the length of thermoelectric leg. a) Peltier cooling mode where the temperature of the solar cell is lower than that of the heat sink; b) Heat pumping mode where the solar cell temperature is higher than that of heat sink; c) Special case of heat pumping where the temperature difference across the thermoelectric device is 0 ; d) Selfpowered heat pumping where the temperature of solar cell is higher than that of heat sink with its input terminals short-circuited. 
Table 1. The net power obtained from the power gained in the solar cell and the power lost in the thermoelectric device for four operating modes. The results were calculated based on a typical low concentration system of $4 \mathrm{x}$, which produces a solar energy intensity of $Q_{i r}=4000 \mathrm{~W} / \mathrm{m}^{2}$. It is assumed that the solar cell has an active area of $5 \mathrm{~cm} \times 5 \mathrm{~cm}$, conversion efficiency of $20 \%$ and temperature coefficient of $-0.5 \% K^{-1}$ and the thermoelectric device has a $Z \bar{T}=1$.

\begin{tabular}{|c|c|c|c|c|}
\hline Power & $\begin{array}{c}\begin{array}{c}\text { Peltier Cooling } \\
\left(\mathbf{T}_{1}<\mathbf{T}_{2}\right)\end{array} \\
\mathrm{T}_{1}=295 \mathrm{~K} \\
\mathrm{~T}_{2}=300 \mathrm{~K}\end{array}$ & $\begin{array}{l}\text { Heat Pumping } \\
\left(\mathbf{T}_{\mathbf{1}}=\mathrm{T}_{\mathbf{2}}\right) \\
\mathrm{T}_{1}=300 \mathrm{~K} \\
\mathrm{~T}_{2}=300 \mathrm{~K}\end{array}$ & $\begin{array}{c}\text { Heat Pumping } \\
\left(\mathbf{T}_{\mathbf{1}}>\mathbf{T}_{\mathbf{2}}\right) \\
\mathrm{T}_{1}=305 \mathrm{~K} \\
\mathrm{~T}_{2}=300 \mathrm{~K}\end{array}$ & $\begin{array}{c}\text { Self-powered } \\
\text { heat pumping } \\
\begin{array}{c}\mathbf{T}_{1}>\mathbf{T}_{2} \\
\mathrm{~T}_{1}=305 \mathrm{~K} \\
\mathrm{~T}_{2}=300 \mathrm{~K}\end{array}\end{array}$ \\
\hline Power gain & $\begin{array}{l}0.05 \mathrm{~W} \\
(2.5 \%)\end{array}$ & $\begin{array}{c}0 \mathrm{~W} \\
(0 \%)\end{array}$ & $\begin{array}{c}-0.05 \mathrm{~W} \\
(-2.5 \%)\end{array}$ & $\begin{array}{c}-0.05 \mathrm{~W} \\
(-2.5 \%)\end{array}$ \\
\hline Power loss & $\begin{array}{c}-0.82 W \\
(-41 \%)\end{array}$ & $\begin{array}{c}-0.07 \mathrm{~W} \\
(-3.5 \%)\end{array}$ & $\begin{array}{c}-0.022 \mathrm{~W} \\
(-1.1 \%)\end{array}$ & $\begin{array}{r}+0.018 \\
(+0.9 \%)\end{array}$ \\
\hline Net power gain & $\begin{array}{c}-0.77 W \\
(-38.5 \%)\end{array}$ & $\begin{array}{c}-0.07 W \\
(-3.5 \%)\end{array}$ & $\begin{array}{c}-0.072 \mathrm{~W} \\
(-3.6 \%)\end{array}$ & $\begin{array}{c}-0.032 \mathrm{~W} \\
(-1.6 \%)\end{array}$ \\
\hline
\end{tabular}

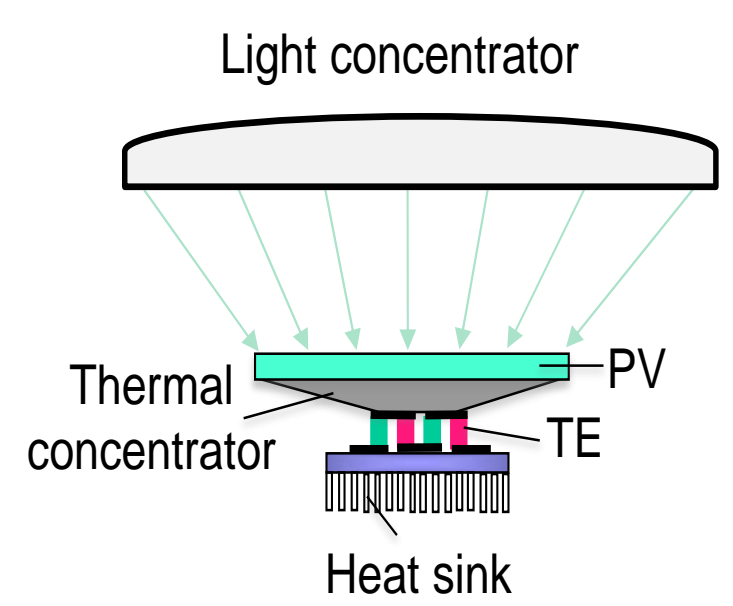

(a)

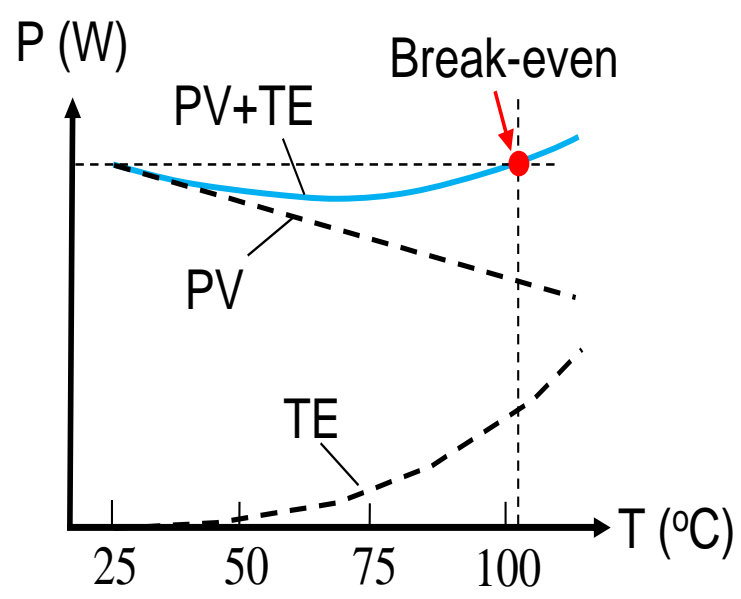

(b)

Fig. 3 a) Schematic tandem PV-TE hybrid system. b) Power output of tandem PV-TE hybrid system as a function of solar cell temperature.

\section{Tandem PV-TE hybrid system}

Solar cells get hot during operation due to thermalisation and absorption. Thermalisation takes place inside solar cells when the charge carriers initially occupying high energy levels jump down to lower energy levels and give off extra energy to crystal lattice, resulting in temperature increase of the solar cells. ${ }^{[2]}$ In addition, the crystal lattice of solar cell materials could absorb long wavelength photons, which can also lead to a temperature increase in solar cells. For solar cells operating under concentrated light, the temperature increase will be more significant. A simple solution to harvest this heat is to mount a thermoelectric generator directly onto the back surface of solar cell as shown in Fig. 3(a). ${ }^{[6]}$ Since the heat flux generated by the solar irradiation is significant less than that required by thermoelectric device, it is usually necessary for a hybrid system to produce sufficient heat flux by using light and/or thermal concentration. However, direct coupling between solar cell and thermoelectric generator as shown in
Fig. 3(a) presents conflicting requirements. For thermoelectric generator to produce large power output, it is necessary to establish a large temperature difference across the thermoelectric generator. This in turn requires the solar cell to operate at high temperature, which will lead to a power reduction in the solar cell as shown schematically in Fig. 3(b). Nevertheless, it is possible to achieve net power gain in such hybrid systems because the power output of the thermoelectric generator increases parabolically with increasing temperature while the power output of the solar cell decreases linearly with the temperature. ${ }^{[7]}$

The feasibility of such hybrid system has been investigated and most of reported work are theoretical simulation with some publications showing significant improvement. However, careful examination of these results reveals that the reported improvement is obtained with a hidden condition the solar cells must operate at extremely high temperature. Clearly, this is an unrealistic requirement because the solar 

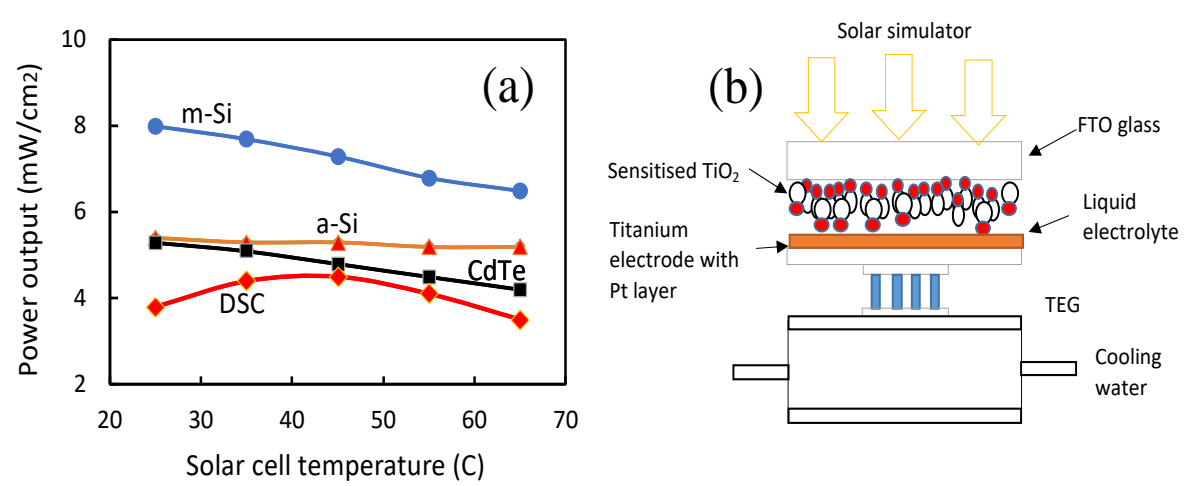

(c)

\begin{tabular}{|c|c|c|c|c|}
\hline & $\begin{array}{c}\mathrm{DSC} \\
\left(\mathrm{T}=25^{\circ} \mathrm{C}\right)\end{array}$ & $\begin{array}{c}\mathrm{DSC} \\
\left(\mathrm{T}=50^{\circ} \mathrm{C}\right)\end{array}$ & $\begin{array}{c}\mathrm{TE} \\
\left(\mathrm{T}_{\mathrm{H}}=50^{\circ} \mathrm{C}\right)\end{array}$ & $\begin{array}{c}\mathrm{DSC}-\mathrm{TE} \\
\left(\mathrm{T}=50^{\circ} \mathrm{C}\right)\end{array}$ \\
\hline $\mathrm{P}_{\max }(\mathrm{mW})$ & 1.06 & 1.11 & 0.09 & 1.20 \\
\hline$\eta(\%)$ & 4.2 & 4.4 & 0.4 & 4.8 \\
\hline
\end{tabular}

Fig. 4 a) Power output per unit area as a function of operating temperature for mono-crystalline silicon (m-Si), amorphous silicon (a-Si), cadmium telluride (CdTe) and dye-sensitized solar cells (DSC). b) Schematic PV-TE hybrid system consisting of DSC and TE. c) The performance of DSC, TE and DSC+TE hybrid system (Reproduced from Ref [8] with permission from Cardiff University).

cells would have been already damaged before reaching the required operating temperatures. Nevertheless, small improvement has been demonstrated experimentally. This was achieved in a hybrid system consisting of dye sensitised solar cell and $\mathrm{Bi}_{2} \mathrm{Te}_{3}$-based thermoelectric generator. ${ }^{\left[{ }^{[3]}\right.}$ The improvement is attributed partly to an unusual temperature dependence of dye-sensitized solar cell. As shown in Fig. 4(a), the power output of the dye-sensitized solar cell increases initially with increasing temperature and then reaches a peak at around $45^{\circ} \mathrm{C}$. This unusual temperature dependence enables the hybrid system to operate at certain higher temperature without causing the power loss from the solar cell. As a result, a net power gain was obtained as shown in Fig. 4(c). Although the improvement in this work is not significant enough for practical application, the work reveals a promising research direction that could lead to a real progress in the development of this type of PV-TE hybrid systems. The key is to discover solar cells that exhibit similar temperature dependence to dyesensitized solar cells, but with higher peak temperature. To date, it has been identified that perovskite and organic solar cells also exhibit similar temperature dependence. The challenge is to shift their peak to much higher temperature (at least beyond $100{ }^{\circ} \mathrm{C}$ ) for such hybrid systems to become economically viable.

\section{Spectral splitting hybrid system}

Solar irradiance has a wide spectrum that covers a wavelength range from $300 \mathrm{~nm}$ to $2500 \mathrm{~nm}$, which can be roughly divided into a visible region $(300 \mathrm{~nm}-750 \mathrm{~nm})$ and an infrared region $(750 \mathrm{~nm}$ to $2500 \mathrm{~nm})$ as shown in Fig. 5(a). A perfect solar cell should be able to convert all photons in the whole solar spectrum into useful energy. In practice, most solar cells can only respond to the part of spectrum, for example, between $300 \mathrm{~nm}$ and $700 \mathrm{~nm}$ by InGaP and perovskite cells, between $300 \mathrm{~nm}$ and $900 \mathrm{~nm}$ by GaAs cells and between 300 and 1000 $\mathrm{nm}$ by Si cells. Multi-junction solar cells have been developed to extend spectral coverage up to $1800 \mathrm{~nm}$ with greatly improved efficiency. ${ }^{\left[{ }^{[9]}\right.}$ The technology required for fabrication of multi-junction cells is expensive and difficult to access.

An alternative approach is to develop spectral splitting PVTE system. ${ }^{[10]}$ It can be seen from Fig. 5(b) that a large amount of solar energy exists in IR spectrum that cannot be used by solar cells (e.g., $42 \%$ in InGaP cells) may be used by thermoelectric generator. Fig. 5(c) shows a possible PV-TE hybrid system based on spectral splitting strategy. Full solar spectrum is split into VR and IR bands using dichroic mirrors. The solar energy in IR band is reflected onto a thermal absorber in the centre to produce heat, while the solar energy in VR band passes through the dichroic mirrors and shine on the solar cells for direct light to electricity conversion. An important advantage of the spectral splitting strategy is that the solar cells are decoupled from the thermoelectric generator. The temperature at the hot side of thermoelectric generator is no longer limited by the operating temperature of the solar cells. Consequently, the thermoelectric generator can operate at a much higher temperature while the solar cells remain at relatively low operating temperature. This facilitates thermoelectric generator to operate at high efficiency condition without causing power reduction in solar cells. Another benefit of spectral splitting is that the solar cells will produce less heat because the heat generation due to infrared absorption has been eliminated and the power loss associated with the temperature increase of the solar cells is minimised. Although involving more complex engineering design, the 


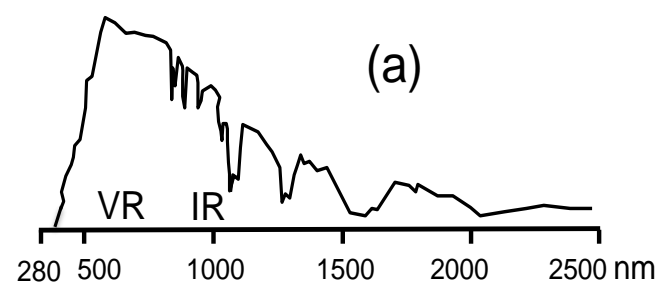

\begin{tabular}{|c|c|c|c|c|}
\hline & C-Si & GaAs & PSC & InGaP \\
\hline Eg (eV) & 1.1 & 1.4 & 1.6 & 1.8 \\
\hline VR & $\sim 77 \%$ & $\sim 61 \%$ & $\sim 60 \%$ & $\sim 58 \%$ \\
\hline IR & $\sim 23 \%$ & $\sim 39 \%$ & $\sim 40 \%$ & $\sim 42 \%$ \\
\hline
\end{tabular}

(b)
$V R+I R$

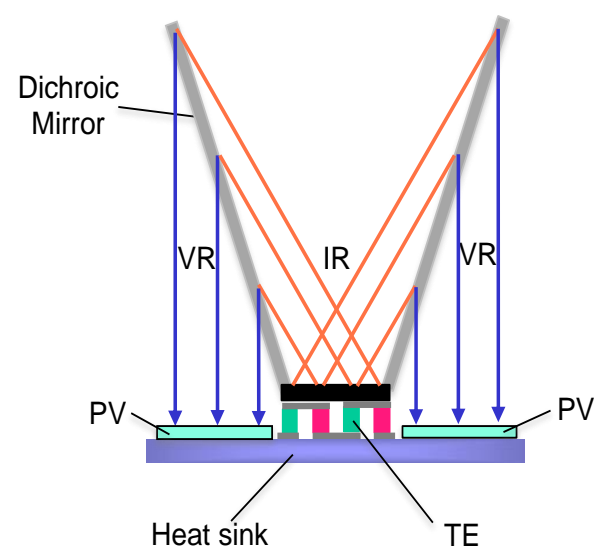

(c)

Fig. 5 a) Solar radiation spectrum consisting of visible (VR) and infrared (IR) bands. b) Solar energy available in VR and IR spectrum based on band gap match of several typical solar cells. c) Schematic PV-TE hybrid system based on spectral splitting approach.

spectral splitting strategy provides the most promising approach for realistic development of high efficiency PV-TE hybrid systems using currently available solar and thermoelectric technologies. A considerable amount of literature has been published on the subject, but they are mostly simulations without experimental study. It was only recently that a detailed experimental investigation has been reported. Although the reported improvement is small, the work demonstrated conclusively that the overall efficiency of a spectral splitting PV-TE hybrid system using a dichroic concentrator is higher than the efficiency of a corresponding PV system that uses an aluminium concentrator. ${ }^{[11]}$ The work also revealed several key challenges that are crucial to development of this hybrid system, which include: efficient light separation and concentration, exact match between dichroic mirror and solar cell, optimal geometry of thermoelectric device, and most importantly, appropriate system design to minimise the thermal energy losses from the system. In addition, the requirement for cooling has implications on economic viability of the system. Watercooling is effective but costly, and hence, developing a costeffective solution for cooling is also important. Recent progress in heat pipe technology may be explored.

\section{Concluding remarks}

PV-TE hybrid systems provide a possible solution for full spectrum solar energy harvesting. Three possible strategies for developing viable PV-TE hybrid system are discussed with a focus on their feasibility and prospect. The use of thermoelectric device for cooling has useful applications in studying the temperature dependence of solar cells or improving their reliability and lifetime. However, the purpose to increase the overall efficiency of a PV-TE hybrid system by thermoelectric cooling is unlikely attainable. Theoretical analysis shows that the power loss from thermoelectric cooling is always higher than the power gain from solar cell. This remains true even if both solar cells and thermoelectric devices reach their respective ultimate efficiencies.

Tandem PV-TE system uses thermoelectric generator to harvest the heat in solar cell by direct coupling of thermoelectric generator to the solar cell. It is a simple and low-cost configuration. However, this type of hybrid system has a major weakness because of conflicting requirements for the operating temperatures of solar cell and thermoelectric generator. As a result, any noteworthy improvement is unlikely with using well-known solar cells, such as Si, GaAs, InGaP, CIGS, and CdTe. Nevertheless, it was discovered that the improvement can be enhanced by using dye-sanitized solar cells owning to their peculiar temperature dependence. Since perovskite and organic solar cells exhibit similar temperature dependence, it is anticipated that some improvement is possible when using these solar cells in a tandem PV-TE system. However, the improvement is likely to be small because the peak temperature of these solar cells is around 50 ${ }^{\circ} \mathrm{C}$. For the tandem hybrid system to be effective, we need to develop new breed of solar cells that can operate at much higher temperature $\left(>100^{\circ} \mathrm{C}\right)$ without power reduction.

Spectral splitting PV-TE system is a promising design that can lead to more significant improvement than tandem PV-TE system and the improvement is readily achievable using currently available solar cells and thermoelectric generators. Since thermoelectric generator is decoupled from solar cell, it can operate at high temperatures and therefore reaches high thermoelectric efficiency without causing power reduction of solar cell. Thus, such system enables more independent design and optimisation of solar cell and thermoelectric generator. To 
date, experimental work has shown some promise of this type of hybrid system and identified key challenges that are crucial to the progress in this direction of research. These include highly effective solar spectral separation and match, high intensity thermal concentration with minimum heat loss, and geometrically optimised thermoelectric generator. In addition, a trade-off between the performance and cost is also important.

\section{Conflict of Interest}

There is no conflict of interest.

\section{Acknowledgement}

The work reported in this paper was carried out with the support of the United Kingdom Engineering and Physical Science Research Council (EPSRC) under the projects of $\mathrm{EP} / \mathrm{K} 029142$ and EP/K022156.

\section{Conflict of Interest}

There is no conflict of interest.

\section{Supporting Information}

Applicable.

\section{References}

[1] P. Singh, N. M. Ravindra, Solar Energy Materials and Solar Cells, 2012, 101, 36-45, doi: 10.1016/j.solmat.2012.02.019.

[2] L. C. Hirst, N. J. Ekins-Daukes, Progress in Photovoltaics: Research and Applications, 2011, 19, 286-293, doi: 10.1002/pip.1024.

[3] F. Crisostomo, R. A. Taylor, D. Surjadi, A. Mojiri, G. Rosengarten, E. R. Hawkes, Applied Energy, 2015, 141, 238-246, doi: 10.1016/j.apenergy.2014.12.044.

[4] H. J. Goldsmid, Electronic refrigeration, Pion, 1986, 198-219. [5] G. Min, N. M. Yatim, Journal of Physics D: Applied Physics, 2008, 41, 2008, 222001.

[6] K. T. Park, S. M. Shin, A. S. Tazebay, H. D. Um, J. Y. Jung, S. W. Jee, M. W. Oh, S. D. Park, B. Yoo, C. Yu, J. H. Lee, Scientific Reports, 2013, 3, 2123, doi: 10.1038/srep02123.

[7] H. Hashim, J. J. Bomphrey, G. Min, Renewable energy, 2016, 87, 458-463, doi: 10.1016/j.renene.2015.10.029.

[8] H. Hashim, Full-spectrum solar energy harvesting using nontechnology enabled photovoltaic/thermoelectric hybrid system, PhD Thesis, Cardiff University, 2015.

[9] H. Cotal, C. Fetzer, J. Boisvert, G. Kinsey, R. King, P. Hebert, H. Yoon, N. Karam, Energy \& Environmental Science, 2009, 2, 174-192, doi: 10.1039/B809257E.

[10] Y. Vorobiev, J. Gonza'lez-Herna'ndez, P. Vorobiev, L. Bulat, Solar Energy, 2006, 80, 170-176, doi: 10.1016/j.solener.2005.04.022.

[11] M. Alnajideen, G Min, Energy Conversion and Management, 2022, 251, 114981, doi: 10.1016/j.enconman.2021.114981.

\section{Author Information}

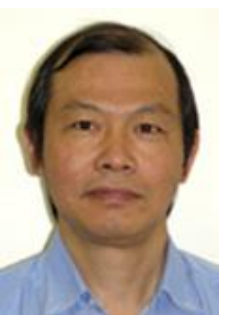

Gao Min is a Professor of Energy Materials, and Group Leader for Magnetics and Materials at School of Engineering, Cardiff University, UK. His research interests focus on fundamental understanding of thermoelectric processes for energy conversion and development of solutionprocessed solar cells and solar energy harvesting systems.

Publisher's Note: Engineered Science Publisher remains neutral with regard to jurisdictional claims in published maps and institutional affiliations. 